\title{
A MARKOV PROCESS WHICH GIVES RISE TO A SEMIGROUP OF EXPANSIVE OPERATORS
}

\author{
T. F. LIN
}

\begin{abstract}
Conditional expectation in a finite complex measure space $(M$, $\mathscr{B}, Q$ ) with $Q(M)=1$ is studied. A Markov process which gives rise to a semigroup of expansive operators is constructed.
\end{abstract}

1. Introduction and notations. A Markov process in a probability space $(M$, $\Re, Q$ ) is always associated with a contraction semigroup (see [2]) because of the fact that $Q$ is a unit positive measure. However, if $Q$ is a finite (signed or complex) measure with $Q(M)=1$, Markov processes can still be defined and can give rise to semigroups of expansive operators. An actual example is contructed in the last section of this article. In order to define Markov processes, conditional expectations must be studied first. This is done in $\$ 2$.

For any measure space $(M, \Re, Q)$, let $|Q|$ denote the total variation of $Q$. If $\mathcal{H}$ is a sub- $\sigma$-field of $\mathscr{B}$, let $Q_{\mathcal{K}}$ denote the restriction of $Q$ to $\mathcal{H}$.

An abstract probability space $(M, \mathscr{B}, Q)$ is a measure space with $|Q|(M)$ $<\infty$ and

$$
Q(M)=1 .
$$

$Q$ will be called an abstract probability measure. A random variable (r.v.) is, in general, a complex-valued $\mathscr{B}$-measurable function on $M$. Because of assumption (1.1), the notion of independence can be discussed. Independent events or random variables are defined as in the case of an ordinary probability space.

Definition. Let $Z$ be a r.v. and $\mathcal{H}$ a sub- $\sigma$-field of $\mathscr{B}$ containing $\sigma(Z) . Z$ is said to be integrable, $\mathscr{H}$-integrable, or absolutely integrable according to whether $|Z|$ is $\left|Q_{\sigma(Z)}\right|$-integrable, $\left|Q_{\mathscr{K}}\right|$-integrable, or $|Q|$-integrable.

2. Conditional expectation. In an ordinary probability space, the conditional expectation $E(Z \mid \mathcal{H})$ of an integrable r.v. $Z$ given a $\sigma$-field $\mathcal{H}$ is an a.e. defined, $\mathcal{H}$-measurable r.v. $Y$ such that

$$
\int_{H} Z d Q=\int_{H} Y d Q
$$

holds for each $H \in \mathcal{H}$. In an abstract probability space, $E(Z \mid \mathcal{H})$ will be defined similarly. However, it should be pointed out that integrability of the

Received by the editors July 17, 1974 and, in revised form, October 22, 1974.

AMS (MOS) subject classifications (1970). Primary 60-00; Secondary 60J25, 28A10.

Key words and phrases. Conditional expectation, $\mathscr{K}$-null set, Markov process, semigroup of operators. 
r.v. $Z$ is not enough, since an $\mathcal{H}$-measurable r.v. $Y$ which satisfies $(2.1)$ might not exist. Even if it does exist, it might not be unique. To overcome these difficulties, we shall introduce the notion of $\mathscr{H}$-null sets. For avoiding the situation that $\infty-\infty$ may appear in the right-hand side of (2.1), the condition that $Z$ be absolutely integrable is too strong and the condition that $Z$ be integrable is too weak. Therefore, we shall impose the integrability condition on $E(Z \mid \mathcal{H})$ instead of on $Z$.

Throughout the rest of this section, let $(M$, $9, Q)$ denote an abstract probability space, $\mathcal{H}$ a sub- $\sigma$-field of $\mathscr{T}$.

Definition. An element $H \in \mathscr{H}$ is called an $\mathfrak{H}$-null set if $\left|Q_{\mathscr{K}}\right|(H)=0$ but $|Q|(H) \neq 0$.

We will let $N_{\mathcal{K}}$ denote the set $\{h=0\}$, where $h$ is the Radon-Nikodym derivative of $Q_{\mathscr{K}}$ with respect to $|Q|_{\mathscr{K}}$. Then $N_{\mathscr{H}}$ is a $\mathscr{K}$-null set which contains every other $\mathcal{K}$-null set $|Q|$-a.e. We will also let $I_{\mathscr{K}}$ denote the indicator function of $N_{\mathscr{K}}^{\mathrm{c}}$, the complement of $N_{\mathscr{H}}$. Note that if $X, Y$ are $\mathcal{H}$-measurable r.v.'s satisfying condition (2.1), then $X=Y|Q|$-a.e. on $N_{\mathcal{K}}^{\text {c }}$. Therefore, there exists at most one $\mathscr{H}$-measurable r.v. $Y$ which satisfies condition (2.1) and vanishes on $N_{\mathcal{K}}$.

Definition. Let $Z$ be a r.v. The conditional expectation of $Z$ given $\mathcal{H}$, denoted by $E(Z \mid \mathcal{H})$, is the $|Q|$-a.e. uniquely defined $\mathcal{H}$-integrable r.v. $Y$, if it exists, which satisfies contition (2.1) and vanishes on $N_{\mathfrak{H}}$.

THeOREM 2.1. Let $Z$ be a r.v. and $\mathcal{H}$ a sub-o-field of $\mathscr{G}$. Suppose that there is a sub- $\sigma$-field $\mathcal{G}$ of $\mathscr{B}$ containing $\sigma(Z) \cup \mathcal{H}$ such that $Z$ is $\mathcal{G}$-integrable. Then $E(Z \mid \mathcal{H})$ exists iff

$$
\int_{H} Z d Q=0
$$

for each $\mathcal{H}$-null set $H$.

Proof. The "only if" part is trivial. To prove the "if" part, let the measure $\lambda$ be defined on $\mathcal{H}$ by

$$
\lambda(H)=\int_{H} Z d Q
$$

Both measures $Q_{\mathcal{K}}$ and $\lambda$ are absolutely continuous with respect to $|Q|_{\mathcal{H}}$. Let $X, Y$ be the Radon-Nikodym derivatives of $Q_{\mathcal{H}}$ and $\lambda$, respectively, with respect to $|Q|_{\mathcal{K}}$; that is

$$
Q_{\mathcal{H}}(H)=\int_{H} X d|Q|, \quad \lambda(H)=\int_{H} Y d|Q|,
$$

for $H \in \mathcal{H}$. From the definition of $N_{\mathcal{K}}$, it is easily seen that $X=0,|Q|$-a.e. on $N_{\mathcal{K}}$, and $X \neq 0,|Q|$-a.e. on $N_{\mathcal{K}}^{\mathrm{c}}$. Let the r.v. $Z^{*}$ be defined by

$$
Z^{*}= \begin{cases}Y / X, & \text { on } N_{\mathscr{H}}^{\mathrm{c}} \\ 0, & \text { on } N_{\mathscr{K}}\end{cases}
$$

Then $Z^{*}$ is $\mathcal{K}$-measurable.

Let $\left\{H_{1}, \ldots, H_{n}\right\}$ be any $\mathcal{H}$-measurable partition of $M$. Then

$$
\begin{aligned}
\sum_{k=1}^{n}\left|\lambda\left(H_{k}\right)\right| & =\sum_{k=1}^{n}\left|\int_{H_{k}} Z d Q\right| \leqslant \sum_{k=1}^{n} \int_{H_{k}}|Z| d\left|Q_{\varrho}\right| \\
& =\int_{M}|Z| d\left|Q_{\varrho}\right|<\infty .
\end{aligned}
$$


This means that $|\lambda|(M)<\infty$. From (2.4), it follows that

$$
\left|Q_{\mathcal{X}}\right|(H)=\int_{H}|X| d|Q|, \quad|\lambda|(M)=\int_{M}|Y| d|Q| .
$$

From (2.2), (2.3) and the second identity of (2.4), we obtain that $Y=0$, $|Q|$-a.e. on $N_{\mathcal{H}}$. Therefore, by (2.5) and (2.6)

$$
|\lambda|(M)=\int_{N_{\mathscr{K}}^{\mathrm{c}}}|Y| d|Q|=\int_{N_{\mathcal{K}}^{\mathrm{c}}}|Z|^{*} d\left|Q_{\mathcal{X}}\right|=\int_{M}|Z|^{*} d\left|Q_{\mathcal{H}}\right| .
$$

Thus $Z^{*}$ is $\mathscr{H}$-integrable. If we can prove that $(2.1)$ holds for $Z^{*}$, then $Z^{*}=E(Z \mid \mathcal{H})$, since $Z^{*}$ is $\mathcal{H}$-integrable and is 0 on $N_{\mathcal{K}}$. However, it is routine to show from (2.2)-(2.5) that (2.1) holds for $Z^{*}$. Hence Theorem 2.1 holds.

REMARK 2.1. A sufficient condition for (2.2) to be true is that $|Q|_{\mathcal{H}}$ and $\left|Q_{\mathcal{X}}\right|$ are equivalent.

REMARK 2.2. There exists an integrable r.v. $Z$ such that (2.2) holds but $E(Z \mid \mathcal{H})$ does not exist. Hence the assumption that $Z$ is $\mathcal{G}$-integrable is necessary.

REMARK 2.3. There exists an example for which $E(Z \mid \mathcal{H})$ exists but is not $\mathcal{G}$-integrable. Therefore, $\mathcal{H}$-integrability is the best we can count on for $E(Z \mid \mathcal{H})$.

COROLlaRy 2.2. Under the hypothesis of Theorem 2.1, we have

$$
\int_{M}|E(Z \mid \mathcal{H})| d\left|Q_{\mathscr{K}}\right| \leqslant \int_{M}|Z| d\left|Q_{\mathcal{G}}\right| \text {. }
$$

COROllary 2.3. Let $\mathcal{H} \subset \mathcal{G}$ be sub- $\sigma$-fields of 93 and $Z$ be a r.v. If condition (2.2) holds for all $\mathcal{H}$-null sets and $E(Z \mid \mathcal{G})$ exists, then $E\{E(Z \mid \mathcal{G}) \mid$ $\mathscr{H}\}=E(Z \mid \mathcal{H})$.

For an integrable r.v. $Z$, let $E(Z)$ denote the integral of $Z$. The following lemma can be obtained by standard procedures (see [1]).

LeMma 2.4. Let $\mathcal{H C}$ be a sub- $\sigma$-field of 9 and $Y, Z$ be r.v.'s.

(i) For constants $a$ and $b, E(a Y+b Z \mid \mathcal{H})=a E(Y \mid \mathcal{H})+b E(Z \mid \mathcal{H})$ if both terms on the right-hand side are well defined.

(ii) If $E(Z \mid \mathcal{H})$ exists and $Y$ is bounded, $\mathcal{H}$-measurabie, then $E(Y Z \mid \mathcal{H})$ $=Y E(Z \mid \mathcal{H})$.

(iii) If $Z$ is bounded and independent of $\mathcal{H}$, then $E(Z \mid \mathcal{H})=E(Z)$.

THEOREM 2.5. Let $Y_{k}, 1 \leqslant k \leqslant n$, be r.v.'s, $\mathcal{K}$ a sub- $\sigma$-field of $\mathscr{B}$ and $f a$ bounded measurable function on $C^{n}$. If there exists an $\mathcal{H}$-measurable r.v. $Z$ such that $\sigma\left\{Y_{k}-Z, 1 \leqslant k \leqslant n\right\}$ is independent of $\mathcal{H}$, then $E\left\{f\left(Y_{1}, \ldots, Y_{n}\right) \mid\right.$ $\mathcal{H}\}$ exists and equals

$$
\left\{E\left[f\left(Y_{1}-Z+z, \ldots, Y_{n}-Z+z\right)\right]\right\}_{z=z} \cdot I_{\mathcal{C}} .
$$

Proof. To prove this, we approximate $f$ by simple functions and use the independence of $\sigma\left(Y_{k}-Z, 1 \leqslant k \leqslant n\right)$ and $\mathcal{H}$ together with Lemma 2.4 and Corollary 2.2 .

3. Construction of a measure. We shall now construct a Markov process which gives rise to a semigroup of expansive operators. Let $p \in R$ and 
$f(t, r)=\exp \left\{-t p r-t r^{2} / 2\right\}, 0 \leqslant t, r \in R$. If we let

$$
g(t, x)=(2 \pi)^{-1} \int_{R} f(t, r) \exp \{-i r x\} d r \quad \text { for } x \in R, t>0
$$

then $g(t, \cdot)$ is an abstract probability density function and

$$
\left\{\begin{array}{l}
g(t, x)=(2 \pi t)^{-1 / 2} \exp \left\{i p x+t p^{2} / 2-x^{2} / 2 t\right\}, \\
\int_{R} g(t, x) d x=f(t, 0)=1, \\
g(t+s, x)=g(t) * g(s)(x)=\int_{R} g(t, x-y) g(s, y) d y .
\end{array}\right.
$$

We will adopt the convention

$$
\int_{A} g(0, x) d x= \begin{cases}0, & \text { if } 0 \notin A \\ 1, & \text { if } 0 \in A .\end{cases}
$$

For each $n \geqslant 1,0 \leqslant t_{1} \leqslant \cdots \leqslant t_{n} \leqslant T$ (some constant), define an $n$ dimensional abstract probability distribution on $R^{n}$ by

$$
F\left(t_{1}, \ldots, t_{n} ; d x_{1}, \ldots, d x_{n}\right)=\prod_{k=1}^{n} g\left(t_{k}-t_{k-1}, x_{k}-x_{k-1}\right) d x_{k}
$$

where $t_{0}=0$ and $x_{0}=0$. Because of (3.1), it is clear that this family of finite-dimensional distributions satisfies the consistency condition. Therefore (see [3, pp. 107-111]), there exists a countably additive set function $P^{*}$ on (D) ${ }^{*}$, the class of all cylinder subsets of $\Omega^{*}=R^{[0, T]}$, such that $P^{*}(D)$ is given by (3.3) for each cylinder set $D$ with coordinates $t_{1} \leqslant \cdots \leqslant t_{n}$ and base $\left(d x_{1}, \ldots, d x_{n}\right)$. For any $A \in \mathscr{B}\left(R^{n}\right)$, the Borel field of subsets of $R^{n}$, it is not hard to see that

$$
\left|\int_{A} F\left(t_{1}, \ldots, t_{n} ; d x_{1}, \ldots, d x_{n}\right)\right| \leqslant \exp \left\{T p^{2} / 2\right\} .
$$

Therefore, $P^{*}$ can be extended to the $\sigma$-field $\mathscr{F}^{*}$ generated by $\mathscr{D}^{*}$ and $\left(\Omega^{*}\right.$, $\left.\mathscr{F}^{*}, P^{*}\right)$ becomes an abstract probability space. Let $y^{*}\left(t, \omega^{*}\right)=\omega^{*}(t), 0 \leqslant t$ $\leqslant T, \omega^{*} \in \Omega^{*}$. Then $y^{*}(0)=0,\left|P^{*}\right|$-a.e., since every cylinder subset of $\left\{y^{*}(0) \neq 0\right\}$ has zero $P^{*}$-measure according to (3.3) and (3.2). The finitedimensional distribution function of $\left\{y^{*}(t)\right\}$ is given by (3.3). We see that $\left\{y^{*}(t)\right\}$ has stationary independent increments.

Let $0 \leqslant s<t \leqslant T, A \in \mathscr{B}(R), G=\left\{y^{*}(t)-y^{*}(s) \in A\right\}$. Then it is easily checked that

$$
\left|P^{*}\right|(G) \leqslant 4 \sup _{D \in \mathcal{D}}\left|P^{*}(G D)\right| \leqslant 4 \exp \left(T p^{2} / 2\right) \int_{A} n(t-s, x) d x
$$

where $n(t, x)$ is the normal density function with mean zero and variance $t$. Hence

$$
\int_{\Omega^{*}}\left|y^{*}(t)-y^{*}(s)\right|^{4} d\left|P^{*}\right| \leqslant 4(t-s)^{2} \exp \left(T p^{2} / 2\right)
$$

Let $\Omega=C[0, T]$ and $\mathcal{F}$ be the $\sigma$-field generated by the class $\mathcal{L}$ of all cylinder subsets of $\Omega$. Then inequality (3.4) implies that there exists a $\left|P^{*}\right|$-a.e. defined measurable mapping $\pi$ from $\left(\Omega^{*}, \mathscr{F}^{*}\right)$ to $(\Omega, \mathscr{F})$ (see $[4$, pp. 217-218]) such that 


$$
\pi\left(y^{*}(t)\right)=y^{*}(t), \quad\left|P^{*}\right| \text {-a.e. }
$$

for each $0 \leqslant t \leqslant T$. Let $P$ be the abstract probability measure on $(\Omega, \mathscr{F})$ induced by $P^{*}$. Then $P$ defines the same finite dimensional distributions on $\mathscr{D}$ as $P^{*}$ does on $\mathscr{D}^{*}$ because of (3.5).

THEOREM 3.1. $(\Omega, \mathscr{F}, P)$ is an abstract probability space such that $y(t, \omega)=\omega(t), \omega \in \Omega, 0 \leqslant t \leqslant T$, is a process starting at 0 with stationary independent increments. The finite-dimensional distribution function of $\{y(t)\}$ is given by (3.3).

Let us now show that $\{y(t)\}$ is a Markov process in the sense that

$$
E\left\{I_{A}(y(t)) \mid \mathscr{F}_{s}^{r}\right\}=E\left\{I_{A}(y(t)) \mid \mathscr{F}_{s}\right\}, \quad|P| \text {-a.e. }
$$

for $0 \leqslant r \leqslant s \leqslant t \leqslant T$, where $\mathscr{F}_{s}=\sigma(y(s)), \mathscr{F}_{s}^{r}=\sigma(y(u), r \leqslant u \leqslant s)$ and $I_{A}$ is the indicator function of a Borel set $A \in \mathscr{G}(R)$. To this end, let $W$ denote the Wiener measure on $(\Omega, \mathscr{F})$.

We make the following observation.

Lemma 3.2. $P$ and $W$ are equivalent. The Radon-Nikodym derivative of $P$ with respect to $W$ is $\exp \left\{i p y(T)+T p^{2} / 2\right\}$.

Corollary 3.3. Let $0 \leqslant s \leqslant t \leqslant T, \mathscr{H}=\mathscr{F}_{t}$ or $\mathscr{F}_{t}$. Then $|P|_{\mathscr{X}}$ and $\left|P_{\mathcal{H}}\right|$ are equivalent and $I_{\mathcal{C}}=1,|P|$-a.e.

THEOREM 3.4. The process $y(t), 0 \leqslant t \leqslant T$, is a Markov process in the sense of (3.6).

Proof. From Corollary 3.3 and Remark 2.1, condition (2.2) is always satisfied. Thus, conditional expectations of both sides of (3.6) exist. Let $\mathscr{H}=\mathscr{F}_{s}$ or $\mathscr{F}_{s}^{r}$ and apply Theorem 2.5; we have

$$
E\left\{I_{A}(y(t)) \mid \mathcal{H}\right\}=\int_{A} g(t-s, x-y(s)) d x \cdot I_{\mathcal{K}} .
$$

Again, by Corollary 3.3, $I_{\mathscr{K}}=1,|P|$-a.e. Hence (3.6) holds. This proves Theorem 3.4.

Corollary 3.5. Let $B$ denote the Banach space, with sup-norm, of all bounded, measurable, complex-valued functions on $R$ and let $S_{t}, 0 \leqslant t \leqslant T$, denote the semigroup of operators on $B$ associated with the Markov process $\{y(t)\}$. Then $\left\{S_{t}\right\}$ is a noncontraction semigroup and $\left\|S_{t}\right\|=\exp \left\{t p^{2} / 2\right\}$, $0 \leqslant t \leqslant T$.

\section{BiBLIOGRAPHY}

1. K. L. Chung, A course in probability theory, Harcourt, Brace \& World, New York, 1968. MR 37 \#4842.

2. E. B. Dynkin, Markov processes, Fizmatgiz, Moscow, 1963; English transl., Vol. I, Die Grundlehren der math. Wissenschaften, Band 121, Academic Press, New York; Springer-Verlag, Berlin, 1965. MR 33 \# 1886; 33 \# 1887.

3. I. I. Gihman and A. V. Skorohod, Introduction to the theory of random processes, "Nauka", Moscow, 1965; English transl., Saunders, Philadelphia, Pa., 1969. MR 33 \#6689; 40 \#23.

4. K. R. Parthasarathy, Probability measures on metric spaces, Probability and Math. Statist., no. 3, Academic Press, New York, 1967. MR 37 \#2271.

Department of Mathematics, Louisiana State University, Baton Rouge, Louisiana 70803 